\title{
Palliative treatment for malignant gastrointestinal obstruction with peritoneal carcinomatosis: enteral stenting versus surgery
}

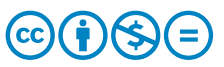

\author{
Authors \\ Meyer $^{1}$, Ryan J. Law ${ }^{4}$, Navtej Buttar ${ }^{1}$ \\ Institutions \\ 1 Division of Gastroenterology and Hepatology, Mayo \\ Clinic, Rochester, Minnesota, United States \\ 2 Division of Radiology, Mayo Clinic, Rochester, \\ Minnesota, United States \\ 3 Government Medical College and Hospital, Chandigarh, \\ India \\ 4 University of Michigan, Gastroenterology and \\ Hepatology, Ann Arbor, Michigan, United States
}

Veeravich Jaruvongvanich ${ }^{1}$, FNU Chesta ${ }^{1}$, Anushka Baruah ${ }^{1}$, Meher Oberoi ${ }^{1}$, Daniel Adamo ${ }^{2}$, Prabh G. Singh ${ }^{3}$, Alyssa

submitted 17.2.2020

accepted after revision 7.7.2020

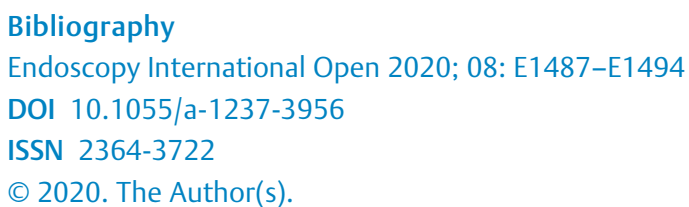

Corresponding author

Navtej Buttar, MD, Division of Gastroenterology and

Hepatology, Mayo Clinic, 200 First Street SW, Rochester, MN 55905, United States

Fax: +1-507-255-6930

Buttar.Navtej@mayo.edu

Supplementary material is available under https://doi.org/10.1055/a-1237-3956

\section{ABSTRACT}

Background and study aims Management of malignant gastrointestinal obstruction ( $\mathrm{MGIO}$ ) is more challenging in the presence of peritoneal carcinomatosis (PC). Outcomes data to guide the management of MGIO with PC are lacking. We aimed to compare the clinical outcomes and adverse events between endoscopic and surgical palliation and identify predictors of stent success in patients with MGIO with PC.

Patients and methods Consecutive inpatients with MGIO with PC between 2000 and 2018 who underwent palliative surgery or enteral stenting were included. Clinical success was defined as relief of obstructive symptoms.

Results Fifty-seven patients with enteral stenting and 40 with palliative surgery were compared. The two groups did not differ in rates of technical success, 30-day mortality, or recurrence. Clinical success from a single intervention (63.2\% versus $95 \%$ ), luminal patency duration ( 27 days vs. 145 days), and survival length (148 days vs. 336 days) favored palliative surgery (all $P<0.05$ ) but the patients in the surgery group had a trend toward better Eastern Cooperative Oncology Group (ECOG) status. The rate of adverse events (AEs) (10.5\% vs. $50 \%)$, the severity of AEs, and length of hospital stay (4.5 days vs. 9 days) favored enteral stenting $(P<0.05)$. The need for more than one stent was associated with a higher likelihood of stent failure.

Conclusions Our study suggests that enteral stenting is safer and associated with a shorter hospital stay than palliative surgery, although unlike other MGIOs, clinical success is lower in MGIO with PC. Identification of the right candidates and potential predictors of clinical success in ECOGmatched large-scale studies is needed to validate these results.

\section{Introduction}

The presence of peritoneal carcinomatosis (PC) portends a poor prognosis and is often seen in patients with advanced and unresectable disease $[1,2]$. It can result from various intra-abdominal and extra-abdominal malignancies, especially ovarian and colorectal cancer $[3,4]$. Malignant seeding within the peritoneal cavity can result in peritoneal nodules, masses, thickening, and bowel wall implantation, which ultimately gives rise to bowel obstruction $[5,6]$. These patients are often initially treated conservatively. However, failure to improve with conserva- 
tive measures is not uncommon, warranting the need for further intervention [2].

The goal of intervention is to relieve the intolerable obstructive symptoms and improve the quality of the patient's remaining life. Palliative surgery and enteral stenting are the two primary treatment modalities. Although surgery can palliate obstructive symptoms $(32 \%-100 \%)$, it leads to frequent serious adverse events (AEs) (7\%-44\%), re-obstruction (6\%-47\%), hospital readmission (38\%-74\%), and reoperation (2\%-15\%), and offers limited survival length (median survival 26-273 days). Most importantly, hospitalization for surgery takes a substantial portion of the patient's remaining life (11\%-61\%) based on a previous systematic review of 17 studies [7]. Enteral stenting is more suitable for patients with advanced malignancy who are too sick to undergo surgery, and it could potentially offer a shorter hospital stay with lower medical costs. However, it is less effective in patients with PC in both upper and lower gastrointestinal obstruction with a significant risk of stent failure from tumor ingrowth, inadequate expansion, clogging, or migration [8-11]. Having predictors of treatment success to identify ideal candidates for enteral stenting is, therefore, critical. While several studies have compared the outcomes between enteral stenting and palliative surgery in the treatment of malignant gastrointestinal obstruction (MGIO), but none have specifically addressed patients with PC [12-14].

Our primary aim was to assess the treatment response patterns of enteral stenting and palliative surgery for MGIO and PC and compare their clinical outcomes and AEs in this most extensive series to date. Our secondary aim was to identify predictors of clinical success after enteral stent placement.

\section{Patients and materials}

This study was a retrospective study of a prospectively collected database from Mayo Clinic, Rochester (Minnesota, United States), and was approved by the Mayo Clinic Institutional Review Board (IRB 14-000586) and was conducted in accordance to Declaration of Helsinki and Strengthening the Reporting of Observational Studies in Epidemiology (STROBE) guidelines.

\section{Study population}

Patients with symptomatic MGIO secondary to unresectable metastatic cancer with PC who were hospitalized and underwent enteral stent placement or palliative surgery from January 2000 to December 2018 were included. MGIO was diagnosed clinically and confirmed by imaging and/or endoscopy. All patients had pathologically proven malignancy and were not candidates for surgical curative treatment. Patients who did not have PC at the time of bowel obstruction; had an insufficient medical record; had no follow-up data of more than 60 days after the first intervention, or had no research authorization were excluded from the study. Baseline and cancer characteristics including age, gender, race, body mass index (BMI), ascites, Eastern Cooperative Oncology Group (ECOG) scale, cancer types, histologic grades, receipt of chemotherapy and radiotherapy, details of gastrointestinal obstruction, and treatmentrelated data were collected from our electronic medical record system. All patients were followed until their last clinic visit or death by chart review for procedure-related AEs and luminal re-obstruction.

The type of bowel obstruction was classified into the extrinsic obstruction and extrinsic obstruction with luminal invasion. An extrinsic obstruction was defined as the obstruction due to an extrinsic compression without invasion. The degree of obstruction was categorized into partial and complete obstruction based on imaging and/or endoscopic findings. The length of obstruction was obtained from computed tomography and/ or fluoroscopy or endoscopic reports. If these data were not reported, the images were interpreted by our radiologist (D.A.), who was blinded to the treatment and patient outcomes. PC was confirmed by imaging and/or cytology/histology. The severity of the PC was assessed based on CT before the intervention and was classified into three categories. Mild carcinomatosis was defined as mild peritoneal thickening with or without sub-centimeter nodules. Moderate carcinomatosis was defined as presence of large discrete peritoneal nodules $>1 \mathrm{~cm}$. Severe carcinomatosis was defined as presence of bowel infiltration. Cancer types were classified into urogynecological cancers (bladder, ovarian, fallopian tubes, endometrium, and cervix) and non-urogynecological cancers. Data regarding the presence of diverticulosis in the same bowel segment of stent placement were also collected from endoscopy reports, as this posed additional challenges to successful stenting. The site of obstruction was classified into gastric outlet, small bowel, right colon (right colon and transverse colon), left colon (left colon and rectum), multiple sites, and anastomotic site. Prior abdominal surgery was any intra-abdominal surgery that involved bowel resection and/or anastomosis.

\section{Procedures}

The treatment decision between endoscopic stent placement and surgery was determined by a consensus among a multidisciplinary care team, including gastroenterologists, medical oncologists, surgeons, and the palliative care team. For endoscopic stent placement, the type and size of stent used were chosen based on the stenosis characteristics. The stent was deployed across the site of obstruction using a standard approach of a guidewire and fluoroscopic guidance. In cases with longsegment obstruction, multiple overlapping stents were placed to ensure the entire length of obstruction was treated. For palliative surgery, resection with primary anastomosis was attempted if possible. Other surgical options included diverting ostomy or bypass surgery based on surgeons' decisions.

\section{Definitions and assessment of clinical outcomes}

Technical success for patients undergoing enteral stent placement was defined as successful deployment of the stent across the stricture site and confirmation of patency by post-intervention imaging using contrast. Technical success for patients undergoing palliative surgery was defined as an absence of bowel leak or postoperative wound dehiscence within the first week. Early clinical success was defined as relief of obstructive symptoms within the first week after a single intervention. Overall clinical success was defined as relief of obstructive symptoms 
- Table 1 Baseline characteristics

\begin{tabular}{|c|c|c|c|}
\hline Characteristics & $\begin{array}{l}\text { Stent group } \\
(n=57)\end{array}$ & $\begin{array}{l}\text { Surgery group } \\
(n=40)\end{array}$ & $P$ value \\
\hline Female [n (\%)] & $39(68.4)$ & $28(70.0)$ & 0.89 \\
\hline - Age [years, mean \pm SD] & $60.4 \pm 13.6$ & $60.7 \pm 15.0$ & 0.93 \\
\hline - Race [caucasian, mean \pm SD] & $54(98.2)$ & $39(97.5)$ & 0.35 \\
\hline - BMI (kg/m²) & $24.7 \pm 5.9$ & $26.0 \pm 6.4$ & 0.35 \\
\hline - Ascites [n (\%)] & 45 (78.9) & $29(72.5)$ & 0.46 \\
\hline Prior abdominal surgery [n (\%)] & $16(28.1)$ & $11(27.5)$ & 0.95 \\
\hline \multicolumn{3}{|l|}{$\operatorname{ECOG}[\mathrm{n}(\%)]$} & 0.06 \\
\hline . $0-1$ & $26(45.6)$ & $26(65.0)$ & \\
\hline . $2-4$ & $31(54.4)$ & $14(35.0)$ & \\
\hline Histologic grade [high grade, n (\%)] & $48(84.2)$ & $35(87.5)$ & 0.65 \\
\hline \multicolumn{3}{|l|}{ Severity of PC [n (\%)] } & 0.052 \\
\hline - Mild & $40(70.2)$ & $21(52.5)$ & \\
\hline - Moderate & $9(15.8)$ & $7(17.5)$ & \\
\hline - Severe & $8(14)$ & $12(30)$ & \\
\hline Chemotherapy [n (\%)] & $37(64.9)$ & $18(45.0)$ & 0.51 \\
\hline Radiotherapy [n (\%)] & $8(14.0)$ & $5(12.5)$ & 0.83 \\
\hline \multicolumn{3}{|l|}{ Type of cancer [n (\%)] } & 0.14 \\
\hline - Urogynecological & $16(28.1)$ & $17(42.5)$ & \\
\hline - Other cancer & $41(71.9)$ & $23(57.5)$ & \\
\hline \multicolumn{3}{|l|}{ Diagnosis of PC [n (\%)] } & 0.06 \\
\hline - Imaging & $38(66.7)$ & $19(47.5)$ & \\
\hline - Cytology/histology & $19(33.3)$ & $21(52.5)$ & \\
\hline - Single or multiple sites of obstruction [multiple, n (\%)] & $2(3.5)$ & $7(17.5)$ & 0.02 \\
\hline \multicolumn{3}{|l|}{ Site of obstruction [n (\%)] } & $<0.001$ \\
\hline - Gastric outlet obstruction & $9(15.8)$ & 0 & \\
\hline - Small bowel & $2(3.5)$ & $13(32.5)$ & \\
\hline - Right side colon & $10(17.5)$ & $5(12.5)$ & \\
\hline - Left side colon & $30(52.6)$ & $14(35.0)$ & \\
\hline - Multiple sites & $2(3.5)$ & $7(17.5)$ & \\
\hline - Anastomosis & $4(7.0)$ & $1(2.5)$ & \\
\hline Degree of obstruction [complete obstruction, $\mathrm{n}(\%)$ ] & $17(29.8)$ & $7(17.5)$ & 0.17 \\
\hline Obstruction length $(\mathrm{cm})$ & $5.9 \pm 5.1$ & $21.1 \pm 8.6$ & 0.10 \\
\hline Intrinsic or extrinsic obstruction [intrinsic, n (\%)] & $25(44.6)$ & $15(37.5)$ & 0.50 \\
\hline
\end{tabular}

with or without additional rescue stenting within 30 days. Recurrence was defined as luminal restenosis at the same location after achieving clinical success. Luminal patency duration was defined as the interval between initial intervention and luminal restenosis in those who achieved clinical success. Post-proce- dural AEs were assessed within 30 days and classified based on the Clavien-Dindo classification [15]. Severe AEs were defined as grade III-V. Grade III was defined as one that required surgical, endoscopic, or radiological intervention. Grade IV was defined as a life-threatening complication requiring admission to 
an intensive care unit. Grade $V$ was defined as the death of the patient. Length of hospital stay was the time from intervention to hospital discharge.

\section{Statistical analysis}

Data were expressed as mean and standard deviation (SD) for continuous variables with normal distribution or median and interquartile range (IQR) for skewed data and proportions for categorical variables. Continuous data were compared using an unpaired Student's t-test and nonparametric Mann-Whitney U test when appropriate. Categorical data were compared using a Chi-square test or Fisher's exact test when cells had expected counts of less than 5. Subgroup analyses stratified by the site of obstruction and ECOG status were performed. A binary logistic regression analysis was used to investigate potential factors influencing clinical success in the stent group. The Kaplan-Meier method and log-rank test were used for the survival analysis. $P<0.05$ was considered significant. The analysis was performed using SPSS software 21 (SPSS Inc., Chicago, Illinois, United States).

\section{Results}

\section{Patients}

Over the 18 years of study, 203 potentially eligible patients were hospitalized with PC and MGIO and were screened. Of these, 89 patients were excluded, as they were not candidates for any intervention; thereby, received either chemotherapy or palliative care. Seventeen were further excluded, as they did not provide research authorization, had insufficient medical records, or had a follow-up of less than 60 days. Finally, a total of 97 patients met our study criteria and were categorized based on the initial palliative treatment modality for MGIO and PC (stent, $n=57$, surgery $n=40$ ). $>$ Table 1 demonstrates baseline patient and lesion characteristics. The stent group and surgery group were not significantly different in terms of their age, gender, race, BMI, cancer type, histologic grades, receipt of chemotherapy and radiotherapy, and ascites. The severity of PC was trending toward higher severity in the surgery group ( $30 \%$ vs. $14 \%, P=0.052$ ), whereas the ECOG performance status was trending toward a higher severity in the stent group (54.4\% vs. $35 \%, P=0.06$ ). For lesion characteristics, left colon was the most common site of obstruction in both groups. More patients in the surgery group than the stent group had multiple malignant obstructions $(17.5 \%$ vs. $3.5 \%, P=0.02)$. The degree of obstruction and type of obstruction (complete vs. partial) were similar between the two groups. - Table 2 lists the type of primary cancer in both groups. Supplementary Fig. $\mathbf{1}, \mathbf{2}$, and $\mathbf{3}$ show radiologic and endoscopic images of various malignant obstructions and enteral stenting.

\section{Clinical outcomes and AEs}

Technical success rates were comparable between the two groups [87.7\% (50/57) in the stent group and $82.5 \%(33 / 40)$ in the surgery group $(P=0.47)]$. The rate of early clinical success from single intervention was significantly higher in the surgery group than in the stent group [95\% (38/40) vs. $63.2 \%(36 / 57)$,
- Table 2 Type of primary cancer in each group

\begin{tabular}{|c|c|c|}
\hline & $\begin{array}{l}\text { Stent group } \\
(n=57)\end{array}$ & $\begin{array}{l}\text { Surgery group } \\
(n=40)\end{array}$ \\
\hline Unknown cancer & $6(10.5)$ & $2(5.0)$ \\
\hline Pancreatic cancer & $5(8.8)$ & $2(5.0)$ \\
\hline Endometrial cancer & $1(1.8)$ & $1(2.5)$ \\
\hline Hepatobiliary cancer & $4(7.0)$ & 0 \\
\hline Breast cancer & $1(1.8)$ & $3(7.5)$ \\
\hline Esophageal cancer & $1(1.8)$ & $1(2.5)$ \\
\hline Ovarian cancer & $14(24.6)$ & $13(32.5)$ \\
\hline Colorectal cancer & $11(19.3)$ & $8(20.0)$ \\
\hline Primary peritoneum cancer & $1(1.8)$ & $1(2.5)$ \\
\hline Bladder cancer & $1(1.8)$ & $3(7.5)$ \\
\hline Appendiceal cancer & $1(1.8)$ & $2(5.0)$ \\
\hline Carcinoid tumor & $3(5.3)$ & $1(2.5)$ \\
\hline Gastric cancer & $5(8.8)$ & $2(5.0)$ \\
\hline Lung cancer & $1(1.8$ & 0 \\
\hline Small bowel adenocarcinoma & $2(3.6)$ & $1(2.5)$ \\
\hline
\end{tabular}

$P<0.001]$. The overall clinical success rate with initial intervention along with or without rescue stenting was also higher in the surgery group than the stent group [95\% (38/40), vs. 78.9 $\%(45 / 57), P=0.03)$ as nine patients in the stent group had additional stent placement and achieved clinical success. In the surgery group, types of surgery were diverting ostomy ( $n=19$, $47.5 \%)$, resection with primary anastomosis ( $n=19,47.5 \%)$, and bypass surgery $(n=2,5 \%)$. Two patients with clinical failure after surgery were treated with conservative management.

Length of hospital stay was significantly shorter in the stent group (4.5 days vs. 9 days, $P=0.008$ ). The rate of 30 -day mortality was not different between the two groups ( $8 \%$ and $6 \%$, $P=0.96)$. There was a trend for better median survival in the surgery group than the stent group (328.5 days vs. 113 days, log-rank $P=0.06)(\triangleright$ Fig. 1). However, as noted earlier, patients in the surgery group had a trend toward better ECOG status.

The 30-day AE rate was higher in the surgery group than in the stent group $(50.0 \%$ vs. $10.5 \%, P<0.001)$. Based on the Clavien-Dindo Classification, the surgery group also had more serious AEs than the stent group $(P<0.001)$ ( $\vee$ Table 3 ).

In patients with early clinical success with a single intervention, median patency duration was significantly shorter in the stent group ( 23.0 days vs. 154.5 days, $P=0.002$ ) compared to the surgery group. $>$ Table 4 lists outcomes after two treatment modalities.

\section{Subgroup analysis}

After stratifying by site of obstruction, early clinical success rates were significantly lower in the stent group than in the surgery group at the small bowel ( $0 \%$ vs. $92.3 \%, P=0.002)$, left co- 


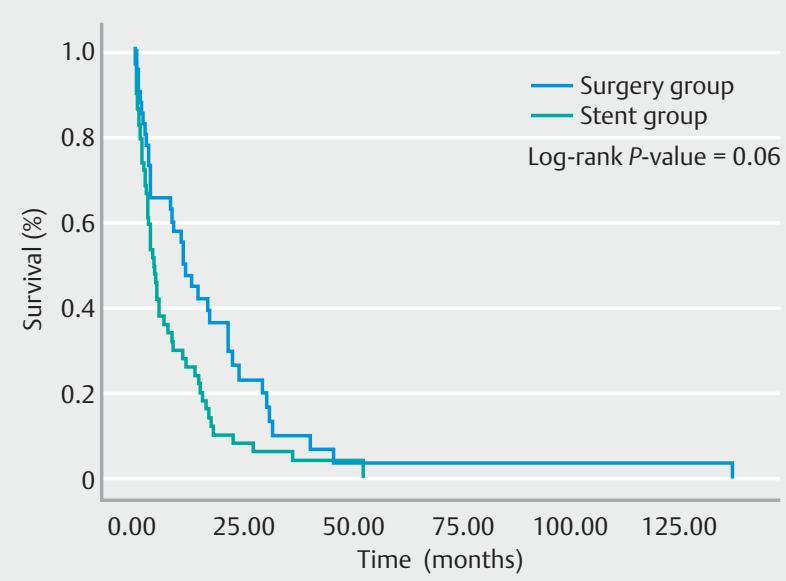

Fig. 1 Survival analysis in the stent group versus the surgery group.

- Table 3 Procedure-related adverse events according to ClavienDindo Classification

\begin{tabular}{|l|l|c|c|}
\hline Adverse events & $\begin{array}{l}\text { Stent group } \\
(\mathbf{n = 6 )}\end{array}$ & $\begin{array}{l}\text { Surgery group } \\
(\mathbf{n = 2 0 )}\end{array}$ & $\boldsymbol{P}$ value \\
\hline Class I & 0 & $4(10.0)$ & $<0.001$ \\
\hline Class II & $3(5.3)$ & $3(7.5)$ & \\
\hline Class III & $3(5.3)$ & $12(30.0)$ & \\
\hline Class IV & 0 & $1(2.5)$ & \\
\hline
\end{tabular}

Ion $(60.0 \%$ vs. $92.9 \%, P=0.026)$, and multiple sites $(0.0 \%$ vs. $100.0 \%, P=0.003$ ) (Supplementary Table 1 ). A subgroup analysis of the rates of early clinical success, overall clinical success, technical success, and AEs and length of hospital stay for different ECOG statuses (0-1 and 2-4) were not different from the main analysis (data not shown). Given the unbalanced distribution of site of obstruction as none in the surgery group had gastric outlet obstruction, a subgroup analysis excluding patients with gastric outlet obstruction was conducted. The rates of early clinical success, technical success, and AEs and length of hospital stay were similar to the primary analysis except that the overall clinical success rate became significantly lower in the stent group than the surgery group $(75.0 \%$ vs. $95.0 \%, P=$ $0.012)$.

\section{Patients with stent failure}

There were 21 patients (36.8\%) with stent failure. The reasons were inadequate stent expansion $(n=7,33 \%)$, embedded stent ( $n=4,19 \%)$, multifactorial $(n=4,19 \%)$, tumor ingrowth $(n=2$, $9.5 \%)$, fecal impaction ( $n=2,9.5 \%)$, migration $(n=1,4.8 \%)$, and inability to pass a guidewire $(n=1,4.8 \%)$, respectively. For management after stent failure, patients were successfully treated with additional stent placement in nine patients (42.9\%) and conservative management in four patients (19\%). Eight patients (38.1\%) underwent palliative surgery.

\section{Prediction of clinical success in the stent group}

Univariate analysis showed that the number of stents used was significantly associated with clinical failure $(P<0.05)$. Despite not being statistically significant, upper gastrointestinal obstruction (vs. lower gastrointestinal) and receipt of chemother-

- Table 4 Clinical outcomes of patients undergoing palliative stenting or surgery for MGIO with PC

\begin{tabular}{|c|c|c|c|}
\hline Outcomes & $\begin{array}{l}\text { Stent group } \\
(n=57)\end{array}$ & $\begin{array}{l}\text { Surgery group } \\
(n=40)\end{array}$ & $P$ value \\
\hline Early clinical success from single intervention [n (\%)] & $36(63.2)$ & $38(95.0)$ & $<0.001$ \\
\hline Overall clinical success [n (\%)] & $45(78.9)$ & $38(95.0)$ & 0.03 \\
\hline Technical success [n (\%)] & $50(87.7)$ & $33(82.5)$ & 0.47 \\
\hline Overall adverse events [n (\%)] & $6(10.5)$ & $20(50.0)$ & $<0.001$ \\
\hline Reobstruction [n (\%)] & $22(38.6)$ & $19(47.5)$ & 0.38 \\
\hline Reobstruction of those with early clinical success [n (\%)] & $14(38.9)$ & $18(47.4)$ & 0.46 \\
\hline Length of hospital stay [days, median (IQR)] & $4.5(2.0-11.0)$ & $9(7-17)$ & 0.008 \\
\hline Luminal patency duration [days, median (IQR)] & $27(9-130)$ & $145(22-552)$ & 0.72 \\
\hline Luminal patency duration in clinical success group [days, median (IQR)] & $23(8-62.5)$ & $154.5(22.8-559.3)$ & 0.002 \\
\hline 30-day mortality [n (\%)] & $8(15.4)$ & $6(15.8)$ & 0.96 \\
\hline Mortality [n (\%)] & $53(93)$ & $36(90)$ & 0.71 \\
\hline Median survival time [days, median (IQR)] & $134(91.7-176.3)$ & $336(224.7-447.3)$ & 0.014 \\
\hline Median survival time in clinical success group [days, median (IQR)] & $148(111.5-184.5)$ & $352(222.7-481.3)$ & 0.002 \\
\hline Duration from intervention to death [days, median (IQR)] & $113(45.8-363)$ & $328.5(83.8-666.8)$ & 0.06 \\
\hline
\end{tabular}


apy were trending toward clinical success. On the other hand, obstruction length, poor preoperative performance status (ECOG 2-4 versus ECOG 0-1), higher severity of PC, and presence of diverticulosis were trending toward clinical failure. Supplementary Table 2 outlines baseline and lesion characteristics of stent success versus stent failure. $>$ Table 5 shows the factors associated with clinical success.

\section{Proposed management algorithm}

Based on the findings of our study, we propose a management algorithm approach to patients with MGIO and PC in > Fig. 2 . First, patients should be evaluated by a multidisciplinary care team for patient candidacy for palliative interventions. If patients are candidates, we suggest an assessment of the obstruction characteristics to guide treatment selection. We recommend considering palliative surgery in patients who have these following unfavorable factors for stent success, including small bowel obstruction, having more than one obstruction site, the need of more than one stent, and the presence of diverticulosis in the same bowel segment of the obstruction. Enteral stenting could then be considered in patients without the factors mentioned above. It should be noted that there are other factors besides the obstruction characteristics for treatment considerations that were not addressed in this study, for example, cost-effectiveness and surgery-related stigma. Our study could not provide guidance on treatment selection for gastric outlet obstruction (GOO) given that there were no patients with $\mathrm{GOO}$ in the palliative surgery group for comparison. However, all patients with GOO in the stent group achieved clinical success.

\section{Discussion}

Identifying a preferred initial intervention for MGIO has been a matter of debate. Both enteral stenting and palliative surgery have their strengths and limitations. While some studies support the use of enteral stenting over surgery to maintain bowel continuity with the avoidance of an ostomy near the end of life $[12,14,16]$, other studies favor surgery $[13,17-19]$. Management is even more challenging in the presence of PC, which negatively impacts patient outcomes, given the advanced stage of malignancy. Our study aimed to provide more insight into short- and long-term outcomes and demonstrate the safety of enteral stenting versus palliative surgery in patients with MGIO and PC. In addition, we also proposed a management algorithm.

Unlike curative surgery, the goals of the palliative intervention are to relieve symptoms with a short hospital stay and low risk of $A E s$, while minimizing the risks of symptom recurrence to improve patient experience and end-of-life quality time outside the hospital. In this study, we found comparable rates of technical success, recurrence, and 30-day mortality between the two treatments. However, early clinical success, overall clinical success, luminal patency duration, and survival length favored palliative surgery while AEs, the severity of AEs, and length of hospital stay favored enteral stenting. It should be noted that there were several baseline differences between the two study
Table 5 Prediction of stent success

\begin{tabular}{|c|c|c|}
\hline & \multicolumn{2}{|l|}{ Univariate analysis } \\
\hline & OR $(95 \% \mathrm{Cl})$ & $P$ value \\
\hline Age & $0.98(0.94-1.03)$ & 0.43 \\
\hline Sex (female) & $1.25(0.39-4.04)$ & 0.71 \\
\hline \multicolumn{3}{|l|}{ ECOG } \\
\hline - $0-1$ & 1 & Ref \\
\hline . $2-4$ & $0.62(0.21-1.84)$ & 0.39 \\
\hline Chemotherapy (yes) & $2.36(0.77-7.28)$ & 0.13 \\
\hline Ascites (yes) & $0.82(0.21-3.16)$ & 0.78 \\
\hline \multicolumn{3}{|l|}{ Severity of PC } \\
\hline - Mild & 1 & Ref \\
\hline - Moderate to severe & $0.54(0.17-1.74)$ & 0.30 \\
\hline \multicolumn{3}{|l|}{ Stent type } \\
\hline - Other types & 1 & Ref \\
\hline - Wallstent & $0.55(0.18-1.66)$ & 0.29 \\
\hline \multicolumn{3}{|l|}{ No. of stent } \\
\hline - 1 & 1 & Ref \\
\hline - $>1$ & $0.20(0.05-0.79)$ & 0.02 \\
\hline \multicolumn{3}{|l|}{ Site of obstruction } \\
\hline - Lower & 1 & Ref \\
\hline - Upper & $2.67(0.65-10.97)$ & 0.17 \\
\hline Obstruction length $(\mathrm{cm})$ & $0.89(0.77-1.03)$ & 0.11 \\
\hline Diverticulosis (yes) & $0.70(0.18-2.77)$ & 0.61 \\
\hline \multicolumn{3}{|l|}{ Type of primary cancer } \\
\hline - Urogynecological & $1.04(0.32-3.43)$ & 0.95 \\
\hline - Other & 1 & \\
\hline $\begin{array}{l}\text { PC, peritoneal carcinomatos } \\
\text { OR, odds ratio }\end{array}$ & astern Cooperative On & Group; \\
\hline
\end{tabular}

groups, including a trending toward higher ECOG status in the stent group, and a trending toward higher severity of PC and longer length of obstruction, and a significantly higher rate of multiple obstruction sites in the surgery group. However, our main goal was to investigate the treatment response pattern after these two modalities in a real clinical setting. To mitigate this, we conducted a subgroup analysis by the site of obstruction. Early clinical success was significantly lower at the small bowel, left colon, and multiple sites, and numerically lower at the right colon and treatment outcomes were similar after exclusion of patients with gastric outlet obstruction. Based on these findings, clinical success appeared lower with enteral stenting at any site of obstruction except for gastric outlet that all patients in our cohort underwent enteral stenting with $100 \%$ clinical success. 


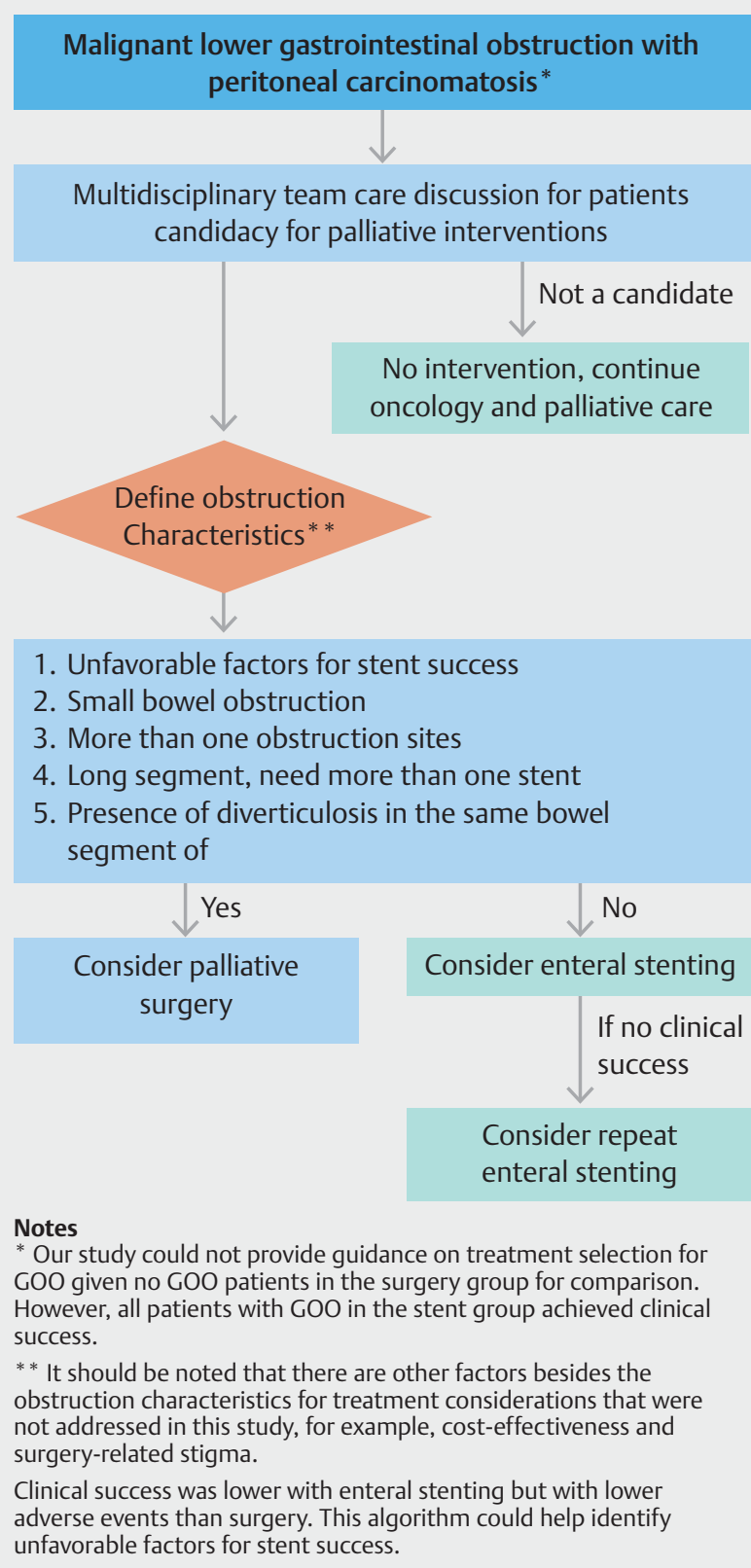

- Fig. 2 Proposed management algorithm for malignant lower gastrointestinal obstruction with peritoneal carcinomatosis.

Regarding poorer performance status in the stent group, it seems to be the practice in many institutions that patients who are too ill to undergo surgery are referred for enteral stenting $[17,19,20]$, and this could contribute to the shorter survival in the stent group. Most importantly, the lower rate of AEs and shorter length of stay despite being performed in poorer performance candidates seem to be the actual benefits of enteral stenting over surgery, but these again need to be interpreted with caution given the baseline differences. The favorable effects of enteral stenting were also seen in other studies that would help achieve the goals of palliation $[12,13,16,17,20]$. Of note, the subgroup analyses for treatment outcomes and
AEs based on different performance status (ECOG 0-1 and ECOG 2-4) were not different from the main analysis. This study could help clinicians make informed decisions in identifying an ideal intervention in each specific clinical scenario. For example, sicker patients should undergo enteral stenting, albeit lower clinical success. Whereas patients with small bowel obstruction or multiple obstruction sites, all of whom had clinical failure after enteral stenting and longer length of obstruction, should undergo surgery, albeit with higher surgical morbidity.

Another advantage of enteral stenting is that it does not preclude surgical intervention or repeat enteral stent placement, as favorable outcomes were demonstrated in our study when the secondary intervention was necessary. In our study, of the 21 patients who had stent failure, eight patients later achieved clinical success with palliative surgery, and additional stent placement was performed in nine patients, all of whom had clinical success afterward. Diverting ostomy (47.5\%) was the most common surgery performed in our surgical cohort. Of note, having an ostomy bag in the surgery group could also lead to a significant stigma to patients toward the end of their lives [21,22]. Despite several strengths of enteral stenting, the clinical success is low, and stent failure is not uncommon, especially with the presence of PC. Therefore, identifying the ideal candidates for stenting is of utmost importance. Need for more than one stent, which is a surrogate of stricture length or severity was significantly associated with stent failure.

Given their effect size, the receipt of chemotherapy and upper gastrointestinal obstruction (versus lower gastrointestinal obstruction) were trending toward stent success, whereas obstruction length, poor ECOG status, and the presence of diverticulosis were trending toward stent failure despite none of these factors achieving statistical significance. This could be due to limited sample size. Further investigation in larger cohorts is warranted. Though not explicitly addressed, cost-effectiveness could be another potential disadvantage of enteral stenting as the luminal patency duration appeared to be much shorter than in the surgery group. It should be noted that these interventions are considered high-risk and complex especially being performed in this group of candidates with underlying cancer and related comorbidities. Of all potentially eligible patients, over $40 \%$ appeared not to be procedural candidates.

Our study has several limitations. As with other retrospective studies, there were uncontrollable factors including providers involved in decision-making, the preferences as well as the performance of surgeons and endoscopists, follow-up intervals, and data collection by different investigators, that could give rise to study bias. Given the retrospective nature, this study was also subject to selection bias of patients with a higher surgical risk toward a lesser invasive procedure like enteral stenting as the treatment decision was made based on patient performance status and comorbidities by a multidisciplinary team care. We also observed more patients with higher ECOG status in the stent group. In an attempt to minimize this unbalanced distribution of the baseline performance status, we conducted a subgroup analysis by ECOG status, which demonstrated results similar to the main analysis. However, we should still con- 
sider the results of this study while making educated decisions about stent versus surgery for patients with MGIO and PC to help identify ideal candidates. Finally, our hospital is a tertiary care center, making it subject to referral bias and potentially limiting the generalizability of this study.

\section{Conclusion}

In summary, enteral stenting is safer and able to associated with a shorter hospital stay than palliative surgery in patients with PC and MGIO. Moreover, it does not preclude subsequent surgery or additional stenting procedures. However, clinical success rates for stenting are lower. Potential predictors of stent failure identified in this study should be considered during decision-making, especially in patients with long, complicated strictures for careful candidate selection. Further studies of ECOG-matched large-scale studies are warranted to validate these findings.

\section{Competing interests}

Dr. Buttar is a consultant of Medrobotics and Ovesco. Dr. Law is a consultant of Olympus, America

References

[1] Coccolini F, Gheza F, Lotti M et al. Peritoneal carcinomatosis. World ] Gastroenterol 2013; 19: 6979-6994

[2] Lambert LA, Wiseman J. Palliative management of peritoneal metastases. Ann Surg Oncol 2018; 25: 2165-2171

[3] Fagotti A, Gallotta V, Romano F et al. Peritoneal carcinosis of ovarian origin. World J Gastrointest Oncol 2010; 2: 102-108

[4] Segelman J, Granath F, Holm T et al. Incidence, prevalence and risk factors for peritoneal carcinomatosis from colorectal cancer. $\mathrm{Br}$ J Surg 2012; 99: 699-705

[5] Goodman MD, McPartland S, Detelich D et al. Chemotherapy for intraperitoneal use: a review of hyperthermic intraperitoneal chemotherapy and early post-operative intraperitoneal chemotherapy. J Gastrointest Oncol 2016; 7: 45-57

[6] Raptopoulos V, Gourtsoyiannis N. Peritoneal carcinomatosis. Eur Radiol 2001; 11: 2195-2206

[7] Paul Olson TJ, Pinkerton C, Brasel KJ et al. Palliative surgery for malignant bowel obstruction from carcinomatosis: a systematic review. JAMA Surg 2014; 149: 383-392
[8] Faraz S, Salem SB, Schattner M et al. Predictors of clinical outcome of colonic stents in patients with malignant large-bowel obstruction because of extracolonic malignancy. Gastrointest Endosc 2018; 87: 1310-1317

[9] Lee JE, Lee K, Hong YS et al. Impact of Carcinomatosis on clinical outcomes after self-expandable metallic stent placement for malignant gastric outlet obstruction. PLoS One 2015; 10: e0140648

[10] Park J], Rhee K, Yoon JY et al. Impact of peritoneal carcinomatosis on clinical outcomes of patients receiving self-expandable metal stents for malignant colorectal obstruction. Endoscopy 2018; 50: 11631174

[11] Rademacher C, Bechtler M, Schneider S et al. Self-expanding metal stents for the palliation of malignant gastric outlet obstruction in patients with peritoneal carcinomatosis. World J Gastroenterol 2016; 22: 9554-9561

[12] Abelson JS, Yeo HL, Mao J et al. Long-term postprocedural outcomes of palliative emergency stenting vs stoma in malignant large-bowel obstruction. JAMA Surg 2017; 152: 429-435

[13] Ahn HJ, Kim SW, Lee SW et al. Long-term outcomes of palliation for unresectable colorectal cancer obstruction in patients with good performance status: endoscopic stent versus surgery. Surg Endosc 2016; 30: 4765-4775

[14] Zheng B, Wang X, Ma B et al. Endoscopic stenting versus gastrojejunostomy for palliation of malignant gastric outlet obstruction. Dig Endosc 2012; 24: 71-78

[15] Dindo D, Demartines N, Clavien PA. Classification of surgical complications: a new proposal with evaluation in a cohort of 6336 patients and results of a survey. Ann Surg 2004; 240: 205-213

[16] Roy A, Kim M, Christein J et al. Stenting versus gastrojejunostomy for management of malignant gastric outlet obstruction: comparison of clinical outcomes and costs. Surg Endosc 2012; 26: 3114-3119

[17] Jang S, Stevens T, Lopez R et al. Superiority of gastrojejunostomy over endoscopic stenting for palliation of malignant gastric outlet obstruction. Clin Gastroenterol Hepatol 2019; 17: 1295-1302 e1291

[18] Jeurnink SM, Polinder S, Steyerberg EW et al. Cost comparison of gastrojejunostomy versus duodenal stent placement for malignant gastric outlet obstruction. J Gastroenterol 2010; 45: 537-543

[19] No JH, Kim SW, Lim CH et al. Long-term outcome of palliative therapy for gastric outlet obstruction caused by unresectable gastric cancer in patients with good performance status: endoscopic stenting versus surgery. Gastrointest Endosc 2013; 78: 55-62

[20] Park CH, Park JC, Kim EH et al. Impact of carcinomatosis and ascites status on long-term outcomes of palliative treatment for patients with gastric outlet obstruction caused by unresectable gastric cancer: stent placement versus palliative gastrojejunostomy. Gastrointest Endosc 2015; 81: 321-332

[21] Dabirian A, Yaghmaei F, Rassouli M et al. Quality of life in ostomy patients: a qualitative study. Patient Prefer Adherence 2010; 5: 1-5

[22] Smith DM, Loewenstein G, Rozin P et al. Sensitivity to disgust, stigma, and adjustment to life with a colostomy. J Res Pers 2007; 41: 787-803 